\title{
Dp breakup reaction investigation under specific kinematic configurations at ITS of the Nuclotron
}

\author{
M. Janek ${ }^{1, *}, A . V$. Averyanov $^{2}, E . V$. Chernykh $^{2}, D$. Enache $^{3}, Y u . V$. Gurchin $^{2}, A . Y u$. Isupov ${ }^{2}$, \\ O. Mezhenska ${ }^{4}$, J.-T. Karachuk ${ }^{2,3}$, A.N. Khrenov ${ }^{2}$, D.O. Krivenkov ${ }^{2}$, P.K. Kurilkin ${ }^{2}$, \\ V.P. Ladygin ${ }^{2}$, N.B. Ladygina ${ }^{2}$, A.N. Livanov ${ }^{2}$, S.M. Piyadin ${ }^{2}$, S.G. Reznikov ${ }^{2}$, \\ Ya.T. Skhomenko ${ }^{2,5}, G$. Tarjanyiova ${ }^{1}, A . A$. Terekhin $^{2}, A . V$. Tishevsky $^{2}, T$. Uesaka $^{6}$, and \\ J. Urban $^{4}$ (DSS Collaboration) \\ ${ }^{1}$ Physics Department, University of Zilina, Zilina, Slovak Republic \\ ${ }^{2}$ Joint Institute for Nuclear Research, Dubna, Russian Federation \\ ${ }^{3}$ National Institute for RD in Electrical Engineering ICPE-CA, Bucharest, Romania \\ ${ }^{4} \mathrm{Pavol}$ Jozef Safarik University in Kosice, Slovak Republic \\ ${ }^{5}$ Belgorod State National Research University, Belgorod, Russian Federation \\ ${ }^{6}$ Nishina Center for Accelerator-Based Science, RIKEN, Wako, Japan
}

\begin{abstract}
D p$ breakup reaction is investigated in the energy range 300-500 $\mathrm{MeV}$ at the Internal Target of the Nuclotron using unpolarized and polarized beams by DSS collaboration. The goal of the study is to obtain information about nucleon-nucleon correlations at short distances, non-nucleonic degrees of freedom and relativistic effects. A part of the breakup data at $300 \mathrm{MeV}$ and 400 $\mathrm{MeV}$ has been obtained in kinematics where relativistic effects would have a significant contribution.
\end{abstract}

\section{Introduction}

The spin structure of the $n p$ Short Range Correlations has been investigated via measurements of the tensor analyzing power $A_{y y}$ in deuteron inclusive breakup at JINR. Data analysis has been performed at various energies in wide regions of the longitudinal $x_{F}$ and transverse proton momentum $p_{T}$ [1]. The $A_{y y}$ data have demonstrated the dependence on at least these two internal variables, but the used approach [2] has failed to describe the data. In the vicinity of the Sagara discrepancy the currently known 3NFs contribute by up to $30 \%$ for $d p$ breakup reaction measured at KVI at the energy of $130 \mathrm{MeV}$ [3]. 3NFs improve the description of a part of the data but break the other one. It has been found that relativistic effect contributions are located mainly at backward angles in the $n d$ elastic scattering cross section at $70 \mathrm{MeV}$ [4] and $250 \mathrm{MeV}$ [5], but their contributions are not large enough to fill discrepancy between the experimental data and theory, even in the case when standard three nucleon forces [6] are used. The next evidence for $3 \mathrm{NFs}$ has been found in binding energies of nuclei with at least four nucleons. Modern NN forces fail to reproduce the data whereas 3NFs significantly improve the description, especially if three pion exchange with $\Delta$ isobar excitation is included [7]. 3NFs in the nucleon scattering was identified in [8] in minimum of the cross section for the $\mathrm{Nd}$ elastic scattering at the energy of $60 \mathrm{MeV}$. The Coulomb effects are located at

\footnotetext{
*e-mail: janek.marian@gmail.com
} 
forward angles and decreasing with increasing of angle. They have negligible contribution in the cross section minima. Generally, relativistic effects are located at the largest angles. Relativistic effects can be also investigated under specific kinematic conditions. Dp breakup reaction at $270 \mathrm{MeV}$ has been investigated in [9] in kinematics where one arm was fixed at $31^{\circ}$ and the second one placed at angles of 28,30 and $32^{\circ}$, respectively. It has been found that 3NFs models TM'99 [6] and Urbana IX [10] gave differing contributions for polarization transfer coefficients $K_{y y}^{y^{\prime}}$ and for $A_{y}, A_{y y}, A_{x x}$ and $A_{x z}$ indicating problems in current models of $3 \mathrm{NFs}$. The $\mathrm{Nd}$ angular distribution and cross section in the region where minima reach and at backward angles are the most studied regions [8, 11, 12]. In general, various 3NFs describe the cross section and polarization observables only in particular cases. The problem can be in the off-shell behavior of $\mathrm{NN}$ interaction [13]. It has been found [14] that the total cross section for the particular cases - exclusive deuteron breakup configurations - is affected only slightly by relativistic effects. For the $d(n, n p) n$ breakup reaction at $200 \mathrm{MeV}$, large relativistic effects have been observed in configuration where one arm is fixed and the second one scans the angular range. The contribution which comes from relativistic effects can reach up to $60 \%$ [14].

The purpose of this study is to investigate possible $3 \mathrm{NFs}$ and relativistic effects in specific configuration using unpolarized and polarized deuteron beam at the energy between $300-$ $500 \mathrm{MeV}$.

\section{Experiment and results}

The goal of the Deuteron Spin Structure (DSS) experimental program is to obtain the information about two and three nucleon forces, including their spin dependent parts, from $d p$ elastic scattering at the energies between $300-2000 \mathrm{MeV}$ and $d p$ breakup reactions with registration of two protons at deuteron energies of $300-500 \mathrm{MeV}$ [15].

Polyethylene and Carbon targets are enclosed in a spherical hull of the Internal Target Station (ITS) [16]. Six various targets can be placed inside the ITS. The $d p$ breakup reaction is investigated by the simultaneous registration of two protons by two detectors operating in coincidence. Up to eight $\Delta E-E$ detectors can be used in the experiment. Details of the $\Delta E-E$ detector construction can be found in [17]. Reasonable agreement was obtained in comparison of energy spectra and missing mass distributions of $d p$ breakup reaction between experiment and GEANT4 simulation which gave us an opportunity to handle with the signal and background in a more flexible way [18]. The stability of amplitude of all photomultiplier tubes (PMTs) was monitored during all the data acquisition period. A detailed description of LED system of PMTs can be found in [17]. Energy resolution of the detector is $\sim 11 \mathrm{MeV}$. Information about the calibration procedure can be found in [19].

In the star condition the momentum vectors of the three outgoing particles have equal amplitudes in the center of mass system and are separated by $120^{\circ}$. In our case two protons are registered. The plane containing the three momentum vectors of the star condition can have various orientation with the respect to the incident beam axis. When the plane is perpendicular to the beam axis, the configuration is known as the space star. When the plane coincides with the beam axis, the configuration is known as the coplanar star. There can be any number of star configurations between these two extremes (intermediate star).

$D p$ breakup reaction simulation has been performed in order to obtain angular distributions of outgoing protons satisfying star configuration at 300, 400 and $500 \mathrm{MeV}$ of deuteron energy. Particular angular configurations were measured at 300 and $400 \mathrm{MeV}$. $D p$ breakup reaction simulation with condition on star kinematics at $400 \mathrm{MeV}$ of deuteron energy is shown in Fig. 1. Subfigures represent the situation when there is no cut on the azimuthal angle be- 
tween detectors (1st plot), azimuthal angle is $90^{\circ}$ (2nd plot), $120^{\circ}$ (3rd plot) and $180^{\circ}$ (4th plot), respectively.

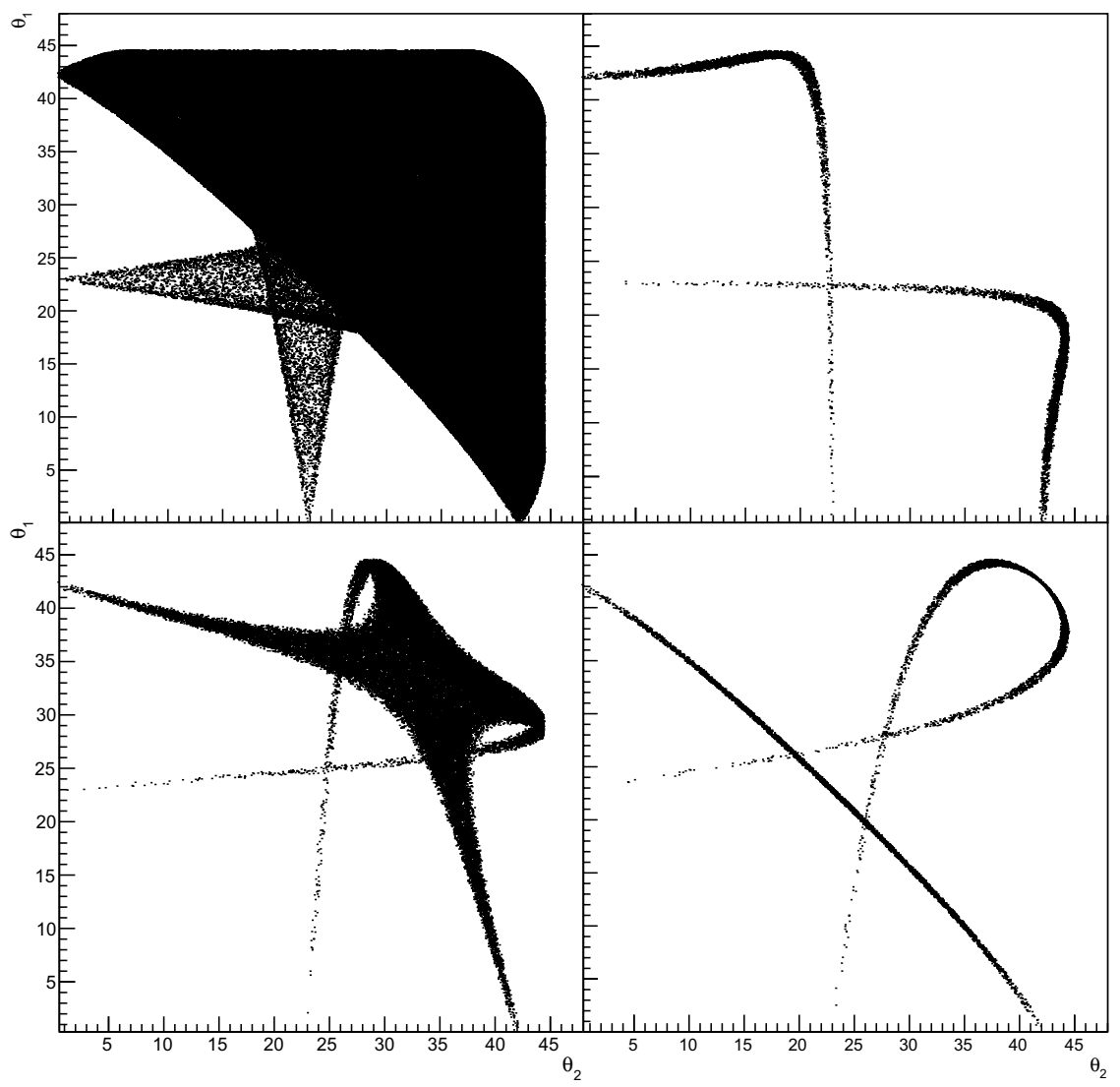

Figure 1. Polar angle configurations $\left(\Theta_{1}\right.$ vs. $\left.\Theta_{2}\right)$ of outgoing protons for the $d p$ breakup reaction at $400 \mathrm{MeV}$ of deuteron energy in case of star kinematics. Subfigures represent the situation when there is no cut on azimuthal angle between detectors (1st plot), azimuthal angle is $90^{\circ}$ (2nd plot), $120^{\circ}$ ( $3 \mathrm{rd}$ plot) and $180^{\circ}$ (4th plot), respectively

In the experiment one arm was fixed at polar angle $\theta_{2}$ of $43.0^{\circ}$ and the second one was placed at angle $\theta_{1}$ of $27.0^{\circ}, 31.0^{\circ}, 35.0^{\circ}, 39.0^{\circ}$ and $43.0^{\circ}$. Energy distribution for the first $E_{1}$ and second $E_{2}$ arms for the case of deuteron energy of $400 \mathrm{MeV}$ is shown in Fig.2. The second arm is fixed at $43^{\circ}$, the first one were positioned at $39^{\circ}, 43^{\circ}$ and $46^{\circ}$. The azimuthal angle between detectors is $180^{\circ}$. Further work was inspired by [9]. Analyzing power $i T_{11}$ at $72.3^{\circ}$ and $76.5^{\circ}$ in $\mathrm{cm}$ was measured under $p p$ quasi conditions. The obtained values at $72.3^{\circ}$ and $76.5^{\circ}$ are $0.10 \pm 0.02$ and $0.11 \pm 0.06$, respectively. The results are in agreement with the world $p p$-elastic scattering data within experimental errors. The values of the vector $i T_{11}$ and tensor $T_{20}$ analyzing powers at polar angles of $34.8^{\circ}$ and $36.8^{\circ}$ and difference in azimuthal angles of $135^{\circ}$ are $0.47 \pm 0.10$ and $0.02 \pm 0.20$ [20]. However, due to low statistics, the large error bars have been obtained. 

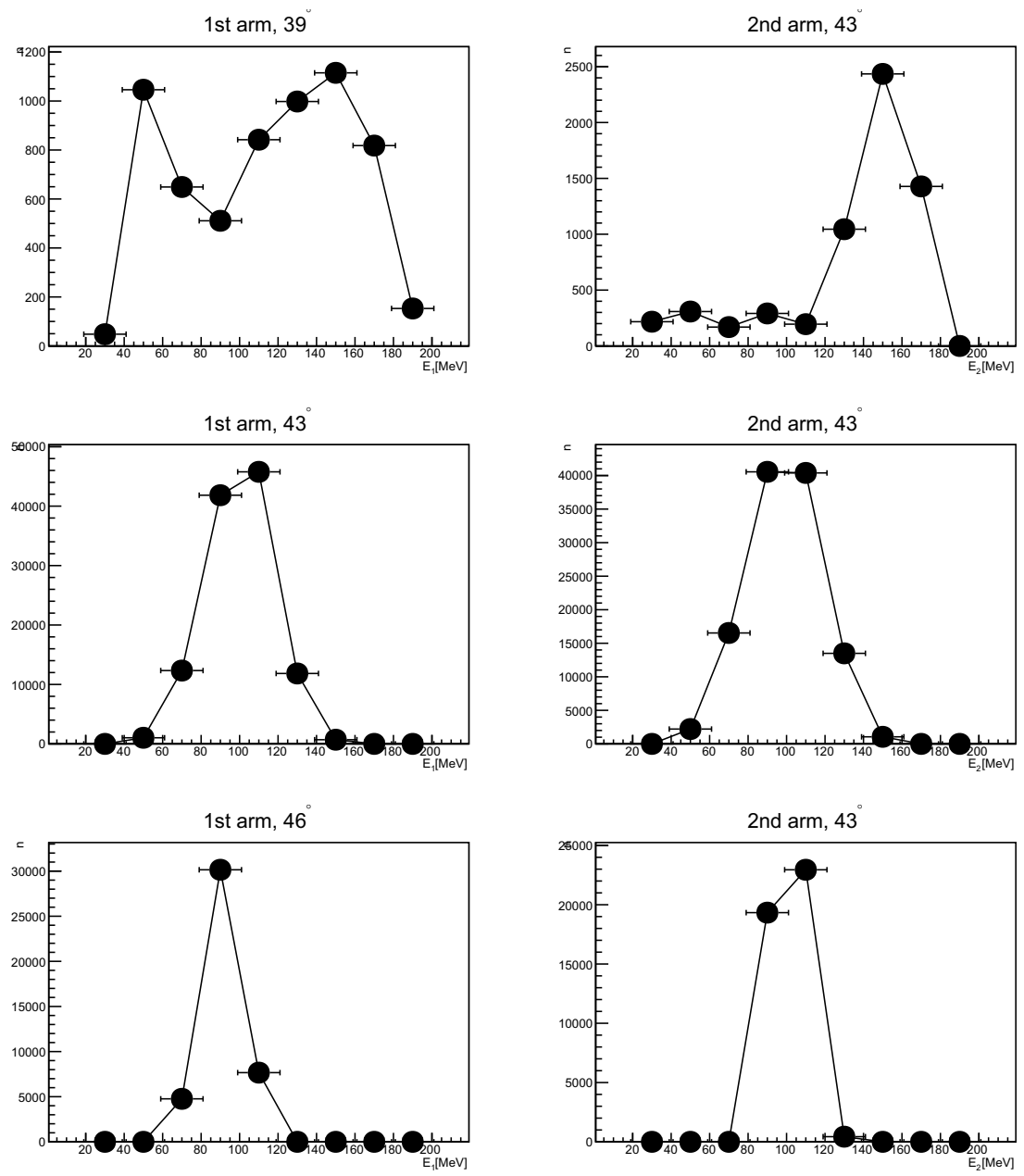

Figure 2. Energy distributions for the first $E_{1}$ and second $E_{2}$ arms for the case of $400 \mathrm{MeV}$ of deuteron energy. The second arm is fixed at $43^{\circ}$, the first one were positioned at $39^{\circ}, 43^{\circ}$ and $46^{\circ}$, respectively. Azimuthal angle between detectors is $180^{\circ}$

\section{Conclusion}

$D p$ breakup reaction has been investigated at intermediate energies using polarized and unpolarized deuteron beams of the Nuclotron. Simulations of $d p$ breakup reaction were performed to find possible angular configurations of detectors. The experimental data have been obtained at 300 and $400 \mathrm{MeV}$ of deuteron energy. Particular data at $400 \mathrm{MeV}$ are presented.

The work has been supported in part by the Ministry of Education, Science, Research, and Sport of the Slovak Republic (VEGA Grant No. 1/0113/18), by the RFBR under grant No. 16-02-00203a and by JINR- Slovak Republic cooperation programs in 2018. 


\section{References}

[1] L. S. Azhgirey et al., Phys. Lett. B 387, 37 (1996)

[2] V. A. Karmanov and A.V. Smirnov, Nucl. Phys. A 546, 691 (1992)

[3] S. Kistryn et al., Phys. Rev. C 72, 044006 (2005)

[4] K. Sekiguchi et al., Phys. Rev. C 65, 034003 (2002)

[5] Y. Maeda et al., Phys. Rev. C 76, 014004 (2007)

[6] S. A. Coon, H.K. Han, Few-Body Syst. 30, 131 (2001)

[7] S. C. Pieper, V. R. Pandharipande, R. B. Wiringa, and J. Carlson, Phys. Rev. C 64, 014001 (2001)

[8] H. Witala, W. Glockle, D. Huber, J. Golak, and H. Kamada, Phys. Rev. Lett. 81, 1183 (1998)

[9] K. Sekiguchi et al., Phys. Rev. C 79, 054008 (2009)

[10] B. S. Pudliner, V. R. Pandharipande, J. Carlson, S. C. Pieper, and R. B. Wiringa, Phys. Rev. C 56, 1720 (1997)

[11] W. Abfalterer W. et al., Phys. Rev. Lett. 81, 57 (1998)

[12] H. Witala, et al., Phys. Rev. C 59, 3035 (1999)

[13] H. Kamada, W. Glockle: Phys. Lett. B 655, 119 (2007)

[14] H. Witala et al., Few-Body Syst 49, 61 (2011)

[15] V. P. Ladygin et al. J. Phys. Conf. Ser. 012131, 295 (2011)

[16] A. I. Malakhov et al., Nucl. Instr. Meth. in Phys. Res. A 440, 320 (2000)

[17] S. M. Piyadin et al., Phys. Part. Nucl. Lett. 8(2), 107 (2011)

[18] M. Janek et al., Particles and Nuclei, Letters 4552 (2014)

[19] M. Janek et al., Particles and Nuclei, Letters 15, 1 (2018)

[20] M. Janek et al., Communications: Scientific letters of the University of Zilina 3, 62 (2017) 